Vol. $42(1990)$ [465-482]

\title{
CONSTANTE RECTANGLE ET BIAIS D'UN ESPACE DE BANACH
}

\author{
JoCELYN DESBIENS
}

We study in this paper the relations existing between Joly's rectangular constant $(\mu)$ and the degree of asymmetry of Birkhoff-James's orthogonality relation $(\beta)$. New bounds on the variation of $\mu$ in terms of $\beta$ and estimation of the values taken by $\beta$ in the case of uniformly convex Banach spaces are given.

\section{Notations}

$(\mathcal{B},\|\cdot\|) \quad$ Espace de Banach réel.

$\mathcal{O} \quad$ Le vecteur origine de l'espace $\mathcal{B}$.

$S(x ; \rho) \quad\{y \in B \mid\|x-y\|=\rho\}, \rho>0$.

$B(x ; \rho) \quad\{y \in \mathcal{B} \mid\|x-y\| \leqslant \rho\}, \rho>0$.

$\mathcal{S} \quad S(\mathcal{O} ; 1)$.

$\mathcal{C}(x, y) \quad$ Le cercle unité du plan $\mathcal{P}$, où $\mathcal{P}$ est le plan contenant les vecteurs indépendants $x$ et $y$ et l'origine $\mathcal{O}$. Donc $\mathcal{C}(x, y)=S \cap \mathcal{P}$.

Nous le noterons par $\mathcal{C}$ lorsque le contexte sera clair.

$\operatorname{dim}(\mathcal{B}) \quad$ La dimension algébrique de $B$.

$[x, y] \quad$ Le segment de droite ayant les vecteurs $x$ et $y$ comme extrémités.

$T_{\gamma} \quad$ L'ensemble des vecteurs propres associés à la valeur propre $\gamma \geqslant 1$

et à l'application $T: X \rightarrow \mathcal{B}$, c'est-à-dire $T_{\gamma}=\{x \in \mathcal{B} \mid T(x)=\gamma x\}$.

$\mathrm{F}(T) \quad$ L'ensemble des points fixes de l'application $T: X \rightarrow \mathcal{B}$. Donc $\mathrm{F}(T)=T_{1}$.

$\mu(\mathcal{B})$ ou $\mu \quad$ La constante rectangle de l'espace $B$.

$\beta(\mathcal{B})$ ou $\beta \quad$ Le biais de l'espace $\mathcal{B}$.

\section{Préllminaires}

Rappelons en premier lieu la définition suivante:

DÉfinition 1: (Birkhoff [3], p.169) Soit $\mathcal{B}$ un espace de Banach et $(x, y) \in \mathcal{B}^{2}$. Nous dirons que $x$ est orthogonal à $y$ au sens de Birkhoff-James, ce que nous noterons par $x \perp_{B J} y$, si et seulement si $\|x+\lambda y\| \geqslant\|x\|, \forall \lambda \in \mathbf{R}$.

Nous aurons besoin du lemme suivant dû à James:

Received 9th January 1990.

Copyright Clearance Centre, Inc. Serial-fee code: 0004-9729/90 \$A2.00+0.00. 
Lemme 1. (James [13], Corollaire 2.2 et Théorème 2.3) Soit $\mathcal{B}$ un espace de Banach. Alors pour tout couple de vecteurs $(x, y) \in \mathcal{B}^{2}$ on a que

(i) $x \perp_{B J} y \Longrightarrow \alpha x \perp_{B J} \beta y, \forall(\alpha, \beta) \in \mathbf{R}^{2}$,

(ii) il existe au moins un couple $(\alpha, \beta) \in \mathrm{R}^{2}$ tel que $x+\alpha y \perp_{B J} y$ et $x \perp_{B J}$ $\beta x+y$.

La définition originale de la relation de Birkhoff-James a été donnée par Birkhoff [3] en 1935. Elle fut reprise un peu plus tard par Fortet [9] et James [13] et utilisée par Joly [15] en 1969 dans sa définition de la mesure de convexité d'un espace de Banach. C'est ce qu'il a dénommé la “constante rectangle” d'un espace vectoriel normé. En voici la définition formelle:

DÉfinition 2: (Joly [15], Définition 2) Soit $\mathcal{B}$ un espace de Banach. On définit la constante rectangle de l'espace $\mathcal{B}$, que l'on dénote $\mu(\mathcal{B})$, par la formule

$$
\mu(\mathcal{B})=\sup _{x \perp_{B} J y} \frac{\|x\|+\|y\|}{\|x+y\|} .
$$

Joly a montré que $\sqrt{2} \leqslant \mu(B) \leqslant 3$. On a en fait la caractérisation suivante (Joly n'a vérifié cette assertion que dans le cas où $\operatorname{dim}(\mathcal{B}) \geqslant 3$ ([15], Proposition, p.307).)

Proposition 1. (Del Rio et Benitez [6], Théorème, p.18) Soit $B$ un espace de Banach. Pour que la norme soit issue d'un produit scalaire il faut et il suffit que $\mu(\mathcal{B})=\sqrt{2}$.

C'est dans le même esprit que nous avons introduit dans l'article [7] une constante associée à un espace de Banach $\mathcal{B}$, que nous notons $\beta(\mathcal{B})$, et dont la définition est:

Définition 3: Soit $\mathcal{B}$ une espace de Banach. On définit le biais de l'espace $\mathcal{B}$, que l'on dénote $\beta(B)$, par la formule

$$
\beta(\mathcal{B})=\sup \left\{\gamma \in \mathbf{R} \mid x+\gamma y \perp_{B J} y ; 0<\|x\| \leqslant\|y\| ; x, y \in \mathcal{B}\right\}
$$

Nous montrerons ci-dessous que ce paramètre peut en fait se redéfinir dans les termes suivants:

DÉfinition 4: (Smith [21], p.225) Soit $\mathcal{B}$ un espace de Banach. On définit la constante de projection métrique de l'espace $\mathcal{B}$, que l'on dénote $\operatorname{MPB}(\mathcal{B})$, par la formule

$$
\operatorname{MPB}(\mathcal{B})=\sup \left\{\left\|P_{\mathcal{E}}\right\| \in \mathbf{R} \mid \mathcal{E} \text { est un sous-espace proximal de } \mathcal{B}\right\}
$$

où

$$
\left\|P_{\varepsilon}\right\|=\sup \left\{\|y\| \in \mathbf{R} \mid y \in P_{\varepsilon}(x),\|x\| \leqslant 1\right\}
$$


et $P_{\mathcal{E}}(x)$ est la projection du vecteur $x$ sur le sous-espace $\mathcal{E}$ de $B$.

Rappelons qu'un sous-ensemble $\mathcal{E}$ d'un espace de Banach $B$ est proximal si, pour tout $x \in B$,

où

$$
\begin{gathered}
P_{\mathcal{E}}(x)=\{y \in \mathcal{B} \mid\|x-y\|=d(x, \mathcal{E})\} \neq \emptyset \\
d(x, \mathcal{E})=\inf \{\|x-y\| \in R \mid y \in \mathcal{E}\}
\end{gathered}
$$

Définition 5: (Baronti [2], p.1076). Soit $B$ un espace de Banach. On définit la constante $\operatorname{MPB}^{\prime}(B)$ associée à l'espace $\mathcal{B}$ par la formule

$$
\operatorname{MPB}^{\prime}(B)=\sup \left\{\left\|P_{\varepsilon}\right\| \in \mathbf{R} \mid \mathcal{E} \text { est un sous-espace dimension } 1 \text { de } B\right\}
$$

DÉfINITION 6: Soit $\mathcal{B}$ un espace de Banach. La projection radiale définie sur $\mathcal{B}$ est la fonction $\rho: B \rightarrow B(\mathcal{O} ; 1)$ donnée par la formule

$$
\rho(x)= \begin{cases}x, & \text { si }\|x\| \leqslant 1 \\ \frac{x}{\|x\|}, & \text { si }\|x\| \geqslant 1\end{cases}
$$

Notons par $k(B)$ la plus petite constante de Lipschitz de la projection $\rho$, c'est-àdire le plus petit nombre réel ayant la propriété que

$$
\|\rho(x)-\rho(y)\| \leqslant k(B)\|x-y\|
$$

et ce pour tout $x, y \in \mathcal{B}$. Les majorants donnés par Dunkl et Williams [8] et Massera et Schäffer [17] montrent que ce nombre est bien défini et a une valeur comprise entre 1 et 2. De plus, DeFigueiredo et Karlovitz [5] ont montré que la relation d'orthogonalité de Birkhoff-James définie sur un espace de Banach $\mathcal{B}$ est symétrique si et seulement si $k(B)=1$.

Proposition 2. (Thele [22], p.483) Soit $B$ un espace de Banach. Alors

$$
k(B)=\sup \left\{\frac{\|y\|}{\|x-y\|} \in \mathbf{R} \mid x \perp_{B J} y, y \neq \mathcal{O}\right\}
$$

Proposition 3. (Baronti [2], Théorème 5) Soit $\mathcal{B}$ un espace de Banach. On a toujours que

$$
M P B(\mathcal{B})=M P B^{\prime}(B)=k(B)
$$




\section{Propriétés du biais D'Un espace de Banach}

Les résultats suivants sont tirés de l'article [7].

Proposition 4. Soit $B$ un espace de Banach. Alors

(1) $1 \leqslant \beta(B) \leqslant 2$.

(2) $\beta(\mathcal{B})=\sup \left\{\gamma \in R \mid x+\gamma y \perp_{B J} y ; 0<\|x\|=\|y\| ; x, y \in \mathcal{B}\right\}$.

(3) $\beta(B)=\sup \left\{\gamma \in \mathbf{R} \mid x+\gamma y \perp_{B J} y ; x, y \in \mathcal{S}\right\}$.

(4) Si $[x, y] \subset \mathcal{S}$ alors $\beta(B) \geqslant\|x-y\|$.

(5) Supposons que $\operatorname{dim}(\mathcal{B})=2$. Pour que $\beta(\mathcal{B})=2$ il faut et il suffit que le cercle unité $\mathcal{C} \subset \mathcal{B}$ soit un parallélogramme.

Proposition 5. Soit $B$ une espace de Banach. Pour que la relation d'orthogonalité de Birkhoff-James définie dans cet espace soit symétrique il faut et il suffit que $\beta(\mathcal{B})=1$.

EXEMPLE 1. Soit $\mathcal{B}_{r}=\mathbf{R}^{2}$ et $r, s \geqslant 1$ deux nombres réels conjugués, c'est-à-dire que $r^{-1}+s^{-1}=1$. Définissons une norme sur $\mathcal{B}_{r}$ par la formule:

$$
\|(x, y)\|_{r}= \begin{cases}\sqrt[r]{|x|^{r}+|y|^{r}} & \text { si } \operatorname{sgn}(x)=\operatorname{sgn}(y), \\ \sqrt[\varepsilon]{|x|^{2}+|y|^{*}} & \text { si } \operatorname{sgn}(x) \neq \operatorname{sgn}(y) .\end{cases}
$$

Il n'est pas difficile de voir que, pour $r \geqslant 1$, l'orthogonalité de Birkhoff-James est symétrique dans $\mathcal{B}_{r}$. Donc $\beta(\mathcal{B})=1$. Par contre $\mathcal{B}_{r}$ n'est pas hilbertien $(r \neq 2)$.

DÉfinition 7: Soit $\mathcal{B}$ un espace de Banach. Nous dirons que $\mathcal{B}$ a la propriété $\mathcal{P}^{\prime}$ si, pour tout scalaire $\gamma>1$ et pour tout couple de vecteurs $(x, y) \in \mathcal{B}^{2}$, l'implication suivante est vraie:

$$
\|x\| \leqslant\|y\| \Longrightarrow\|x+y\| \leqslant\|x+\gamma y\| .
$$

Proposition 6. Soit $\mathcal{B}$ un espace de Banach, Alors

(1) $\beta(B)=1$ entraine que $B$ possède la propriété $\mathcal{P}^{\prime}$.

(2) Si $\mathcal{B}$ a la propriété $\mathcal{P}^{\prime}$ et que $\beta(\mathcal{B})>1$ alors $\mathcal{B}$ n'est pas strictement convexe.

(3) Le fait que $\mathcal{B}$ est soit strictement convexe et qu'il ait un biais égal à 1 est équivalent au fait que $\mathcal{B}$ possède la propriété $\mathcal{P}^{\prime}$.

Définition 8: Soit $\mathcal{B}$ un espace de Banach, $0<\varepsilon \leqslant 1, x \in \mathcal{S}$ et $z \in S(\mathcal{O} ; \varepsilon)$. Parcourons le segment $[x, z]$ dans le sens de $x$ à $z$ et notons par $y$ le premier point de contact entre le segment $[x, z]$ et la sphère $S(\mathcal{O} ; \varepsilon)$. Nous dirons alors que $\mathcal{B}$ a la propriété $\mathcal{P}^{\prime \prime}$ si, quel que soit le choix du rayon $\varepsilon$ et des vecteurs $x$ et $z$, l'inégalité

$$
\|x-y\| \leqslant 1
$$


est toujours vérifiée.

Géométriquement, la propriété $\mathcal{P}^{\prime \prime}$ signifie que, pour un observateur placé en un point $x$ de la sphère unité $S$, tous les points visibles de la sphère $S(O ; \varepsilon)(0<\varepsilon \leqslant 1)$ appartiennent à la boule $B(x ; 1)$.

Proposition 7: Soit $B$ un espace de Banach.

(1) $\mathrm{Si}$, dans cet espace, la relation d'orthogonalité de Birkhoff-James est symétrique alors cet espace à la propriété $\mathcal{P}^{\prime \prime}$.

(2) Si $\mathcal{B}$ est strictement convexe et s'il possède la propriété $\mathcal{P}^{\prime \prime}$, il possède alors la propriété $\mathcal{P}^{\prime}$.

Proposition 8. Soit $\mathcal{B}$ un espace de Banach strictement convexe. Les conditions suivantes sont alors équivalentes:

(1) La relation d'orthogonalité de Birkhoff-James définie sur $\mathcal{B}$ est symétrique.

(2) $\beta(B)=1$.

(3) $\mathcal{B}$ possède la propriété $\mathcal{P}^{\prime}$.

(4) $\mathcal{B}$ possède la propriété $\mathcal{P}^{\prime \prime}$.

Proposition 9. Soit $B$ un espace de Banach strictement convexe avec $\operatorname{dim}(B) \geqslant 3$. Les conditions suivantes sont alors équivalentes:

(1) La norme sur $\mathcal{B}$ est issue d'un produit scalaire.

(2) La relation d'orthogonalité de Birkhoff-James définie sur $\mathcal{B}$ est symétrique.

(3) $\beta(\mathcal{B})=1$.

(4) $\mathcal{B}$ possède la propriété $\mathcal{P}^{\prime}$.

(5) $\mathcal{B}$ possède la propriété $\mathcal{P}^{\prime \prime}$.

DÉfinition 9: Soit $\mathcal{B}$ un espace de Banach, $X \subset \mathcal{B}$ et $T: X \rightarrow \mathcal{B}$ une application. Nous dirons que $T$ est pseudo-contractante si $\forall(x, y) \in X^{2}$ et $\forall r>0$ on a que

$$
\|x-y\| \leqslant\|(1+r)(x-y)-r(T(x)-T(y))\| .
$$

Proposition 10. Soit $\mathcal{B}$ un espace de Banach strictement convexe de biais égal à 1 et $T: X \rightarrow \mathcal{B}$ une application pseudo-contractante telle que $T_{\gamma} \neq \emptyset$ pour tout $\gamma \in[1, \tau]$ avec $\tau>1$. Il existe alors une application $\phi:] 1, \tau] \rightarrow X$ définie par

$$
\left.\left.\phi(\gamma)=\omega_{\gamma} \Longleftrightarrow \omega_{\gamma} \in T_{\gamma}, \quad \forall \gamma \in\right] 1, \tau\right]
$$

ayant les propriétés suivantes:

(1) $\phi$ est continue sur tout $] 1, \tau]$.

(2) $\gamma \leqslant \mu \Longrightarrow\|\phi(\mu)\| \leqslant\|\phi(\gamma)\|$.

(3) $\sup \{\|\phi(\gamma)\| \mid \gamma \in] 1, \tau]\} \leqslant \inf \{\|x\| \mid x \in F(T)\}$. 
(4) $\mathcal{O} \in X$ et $\gamma \rightarrow \infty \Longrightarrow \phi(\gamma) \rightarrow \mathcal{O}$.

(5) La fonction $h:] 1, \tau] \rightarrow[0, \infty]$ définie par

$$
h(\gamma)=\|\phi(\gamma)-T(\phi(\gamma))\|, \quad \forall \gamma \in] 1, \tau]
$$

est non décroissante.

Proposition 11. (Morales [19]) Soit $B$ un espace de Banach strictement convexe, $X$ un convexe fermé de $B$ et $T: X \rightarrow X$ une application continue et pseudocontractante. Alors $F(T)$ est un convexe fermé de $\mathcal{B}$.

Géométriquement, nous pouvons dire que l'ensemble des vecteurs propres associés aux valeurs propres $\gamma>1$ d'une application continue et pseudo-contractante $T$ forme une courbe de Jordan dans l'ensemble $X \subset B$ originant, si $X$ est un convexe fermé de $\mathcal{B}$, de l'unique point fixe de norme minimale de l'application $T$ et tendant progressivement vers l'origine de l'espace $\mathcal{B}$. Les prochaines propositions (Nous tenons à remercier ici le professeur Dan Amir de l'Université de Tel-Aviv pour les conseils qu'il nous a donnés concernant le lien existant entre les différents paramètres que nous étudions ici.) n'apparaissent pas dans [7].

Proposition 12. Soit $\mathcal{B}$ un espace de Banach. On a toujours que

$$
M P B(B)=M P B^{\prime}(\mathcal{B})=k(\mathcal{B})=\beta(\mathcal{B})
$$

DÉmonstration: Au vu de la Proposition 3, il ne reste qu'à démontrer la validité de l'égalité de droite. Soit donc $x$ et $y$ deux vecteurs de $\mathcal{S}$. On peut trouver un nombre réel $\gamma$ tel que $x+\gamma y \perp_{B J} y$. Posons $\omega=x+\gamma y$. Alors

$$
\begin{aligned}
\|\boldsymbol{\omega}-\boldsymbol{x}\| & =\|(\boldsymbol{x}+\boldsymbol{\gamma y})-\boldsymbol{x}\| \\
& =\|\boldsymbol{\gamma} \boldsymbol{\|}\| \\
& =|\boldsymbol{\gamma}| \cdot\|y\| \\
& =|\boldsymbol{\gamma}| .
\end{aligned}
$$

Ainsi $|\gamma|$ est égal à la longueur de la projection du vecteur $x$ sur le sous-espace de dimension $1 \mathbf{R} y=\{\lambda y \in B \mid \lambda \in \mathbf{R}\}$. Il suffit maintenant d'appliquer la Proposition 3.

Une conséquence immédiate de cette proposition est que, pour tout espace de Banach $\mathcal{B}$, on a $\beta(B)=\beta\left(\mathcal{B}^{*}\right)$, où $\mathcal{B}^{*}$ est le dual topologique de $\mathcal{B}$ (Franchetti [10], Théorème, p.427). De la même façon, on peut montrer que $B$ est uniformément non carrable (James [14], Définition 1.1) si et seulement si $\beta(B)<2$ (Smith [21], Théorème 2.1). 


\section{Constante rectangle et biais D'un espace de Banach}

\subsection{RéSUltatS PRÉlIMINAIRES}

Soit $\mathcal{B}$ un espace de Banach et $x, y$ deux vecteurs de $\mathcal{B}$. Rappelons auparavant les inégalités suivantes:

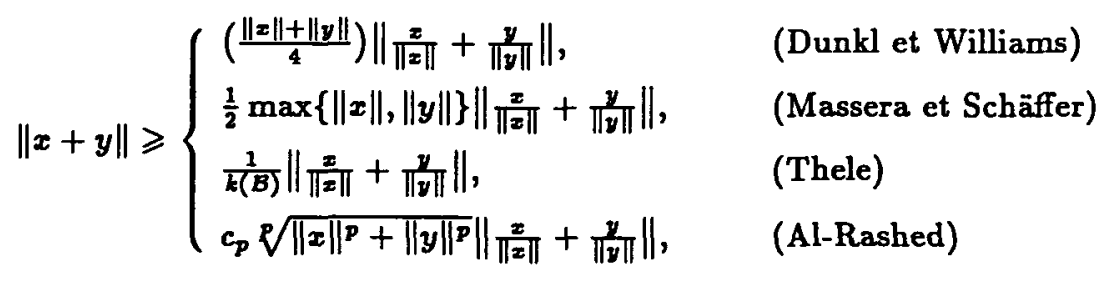

où, dans le dernier cas, $c_{p}=1 / 4$ si $p \geqslant 1$ et $c_{p}=2^{-1-1 / p}$ si $0<p \leqslant 1$. Ces inégalités sont dues respectivement à Dunkl et Williams [8], Massera et Schäffer [17], Thele [22] et $\mathrm{Al}$-Rashed [1]. II est à noter aussi que l'inégalité de Thele n'est vraie que pour des vecteurs $x, y$ tels que $\|x\| \geqslant 1$ et $\|y\| \geqslant 1$. Les autres inégalités sont vraies cependant pour toutes les valeurs de $x$ et $y$.

Soit donc $x$ et $y$ deux vecteurs de $\mathcal{S}$ tels que $x+\beta y \perp_{B J} y$, c'est-à-dire tels que le coefficient $\gamma$ associé à ces deux vecteurs atteint sa plus grande valeur. Si tel n'est pas le cas, il est toujours possible de trouver un nombre réel $\gamma=\beta-\varepsilon(\varepsilon>0)$ tel que $x+\gamma y \perp_{B J} y$. Il suffit ensuite d'utiliser des arguments de continuité et de passage à la limite pour arriver aux résultats désirés. Par mesure de simplicité, nous assumerons tout au long de ce chapitre l'existence de tels vecteurs $x$ et $y$.

Proposition 13. Soit $x, y \in \mathcal{S}$ deux vecteurs tels que $x+\beta y \perp_{B J} y$. Alors

(1) $\|x+\beta y\| \leqslant \mu-\beta$.

(2) $\|x+y\| \leqslant 1$.

(3) $\mu \geqslant 2 \sqrt{\|x+y\|}$.

(4) $\|x+y\|=1 \Longrightarrow \mu \geqslant 2$.

(5) $\|x+y\|=1 \Longrightarrow B$ n'est pas strictement convexe.

DÉMONSTRATION:

(1) En effet, $\mu \geqslant\|x+\beta y\|+\beta$ car $x+\beta y \perp_{B J} y$ et $\|y\|=1$.

(2) La fonction $\varphi(\lambda)=\|x+\lambda y\|, \forall \lambda \in \mathbf{R}$, est convexe et atteint son minimum absolu au point $\beta \geqslant 1$. Or $\varphi(0)=\|x\|=1$. Si $\varphi(1)=\|x+y\|>1$, il est impossible dans ce cas que $\varphi$ puisse atteindre son minimum au point $\beta$. Contradiction.

(3) On sait que $\|x+\beta y\| \leqslant \mu-\beta$. Appliquant l'inégalité de Thele et la 
Proposition 12 aux vecteurs $x$ et $\beta y(\|\beta y\|=\beta\|y\|=\beta \geqslant 1)$ on obtient

$$
\begin{gathered}
\|x+\beta y\| \geqslant \frac{1}{\beta}\|x+y\| \\
\mu-\beta \geqslant \frac{1}{\beta}\|x+y\| . \\
\mu \geqslant \frac{\|x+y\|}{\beta}+\beta \\
\geqslant 2 \sqrt{\|x+y\|}
\end{gathered}
$$

car la fonction $x \mapsto c / x+x$ atteint son minimum au point $x=\sqrt{c}$.

(4) Conséquence immédiate de la relation précédente.

(5) En effet, le cercle unité $\mathcal{C}(x, y)$ contient dans ce cas le segment de droite $[x, x+\beta y]$

Il est démontré dans Baronti [2], Théorème 3, que la double inégalité suivante est toujours vraie:

$$
2 \beta-1 \leqslant \mu \leqslant \beta+1
$$

L'inégalité de gauche est une conséquence de la relation 13.1. En effet, $\mu \geqslant\|x+\beta y\|+$ $\beta \geqslant \beta\|y\|-\|x\|+\beta=2 \beta-1$ car $\|x\|=\|y\|=1$. Nous verrons un peu plus loin que l'inégalité de droite se déduit aussi d'une relation plus spécifique unissant $\mu$ à $\beta$.

\subsection{Minorant DE la Constante Rectangle}

Nous nous attaquons maintenant au problème consistant à minorer la valeur de $\mu(\mathcal{B})$ en fonction de la valeur de $\beta(B)$. Nous voulons aussi obtenir une meilleure estimation que celle donnée par l'inégalité (1). La Proposition 13.1 affirme que $\| x+$ $\beta y \| \leqslant \mu-\beta$. On sait de plus (Proposition 12) que $\beta(\mathcal{B})=k(\mathcal{B})$ et que

$$
\begin{aligned}
\|x+\beta y\| & \geqslant \begin{cases}\frac{1}{\beta}\|x+y\| & \text { (Thele) } \\
\frac{1}{2} \max \{1, \beta\}\|x+y\| & \text { (Massera et Schäffer) }\end{cases} \\
\text { Mais } \quad\|x+y\| & \leqslant \begin{cases}\beta(\mu-\beta), \quad 1 \leqslant \beta \leqslant \sqrt{2} \\
\frac{2}{\beta}(\mu-\beta), \quad \sqrt{2} \leqslant \beta \leqslant 2 .\end{cases} \\
\mu & \geqslant \frac{\|x+\beta y\|+(\beta-1)}{\|x+y\|} \\
& \geqslant \begin{cases}\frac{1}{\beta}+\frac{\beta-1}{\|x+y\|}, & \text { (Thele) } \\
\frac{\beta}{2}+\frac{\beta-1}{\|x+y\|}, & \text { (Massera et Schäffer) }\end{cases}
\end{aligned}
$$


$\Longrightarrow \quad\|x+y\| \geqslant \begin{cases}\frac{\beta(\beta-1)}{\mu \beta-1}, & 1 \leqslant \beta \leqslant \sqrt{2} \\ \frac{2(\beta-1)}{2 \mu-\beta}, & \sqrt{2} \leqslant \beta \leqslant 2 .\end{cases}$

En conclusion,

$$
\frac{\beta(\beta-1)}{\mu \beta-1} \leqslant\|x+y\| \leqslant \beta(\mu-\beta) \text { et } \frac{2(\beta-1)}{2 \mu-\beta} \leqslant\|x+y\| \leqslant \frac{2}{\beta}(\mu-\beta)
$$

d'où

$$
\begin{aligned}
& \beta-1 \leqslant(\mu-\beta)(\mu \beta-1) \\
& \beta-1 \leqslant(\mu-\beta)\left(2 \frac{\mu}{\beta}-1\right) .
\end{aligned}
$$

Résolvons l'inégalité (2). On peut la réécrire sous la forme

$$
\mu^{2} \beta-\mu\left(1+\beta^{2}\right)+1 \geqslant 0 \text {. }
$$

Ce qui donne

$$
\mu \geqslant \frac{1+\beta^{2}+\sqrt{\left(1+\beta^{2}\right)^{2}-4 \beta}}{2 \beta},
$$

l'autre solution étant impossible $(\mu>1)$. Cette borne inférieure est meilleure que celle de Baronti pour toutes les valeurs de $\beta$ comprises entre 1 et la solution $\beta_{0}$ de l'équation

$$
\Rightarrow \quad \begin{aligned}
2 \beta-1 & =\frac{1+\beta^{2}+\sqrt{\left(1+\beta^{2}\right)^{2}-4 \beta}}{2 \beta} \\
\beta_{0} & =\frac{1+\sqrt{17}}{4} \\
& \approx 1.280776407 \\
& <\sqrt{2} .
\end{aligned} .
$$

Remarquons que, de cette inégalité, on tire

$$
\mu-\beta \geqslant \frac{\mu-1}{\mu \beta},
$$

expression qui est une meilleure estimation du minorant de la valeur de $\mu-\beta$ que celle donnée par l'inégalité (1). Finalement, de l'inégalité (3) on tire

$$
\Rightarrow \quad \mu \geqslant \frac{3 \mu^{2}-3 \beta \mu+\beta \geqslant 0}{4},
$$

l'autre solution étant impossible $(\mu<1)$. Il n'est pas possible d'obtenir dans ce cas un meilleur minorant de $\mu$ car

$$
2 \beta-1 \geqslant \frac{3 \beta+\sqrt{9 \beta^{2}-8 \beta}}{4}
$$

pour tout $1 \leqslant \beta \leqslant 2$. 


\subsection{Majorant de la constante Rectangle}

Montrons en premier que $\mu-\beta \leqslant 1 / \beta$, pour $1 \leqslant \beta \leqslant \sqrt{2}$. Considérons deux cas. Premier cas: $\beta=1$. Alors $\mu \leqslant \beta+1=2$ et donc $\mu-\beta \leqslant 2-1=1 \leqslant 1 / \beta$. Second cas: $\beta>1$. Posons

$$
k \geqslant \frac{\beta(\beta-1)}{\mu \beta-1}
$$

et résolvons pour $\mu$. On a alors

$$
\mu \geqslant \frac{\beta-1}{k}+\frac{1}{\beta}
$$

Posons

$$
\mu_{k}=\frac{\beta-1}{k}+\frac{1}{\beta}
$$

Si $\mu_{k} \geqslant \mu_{k^{\prime}}$ il est possible alors de conclure que $k \leqslant k^{\prime}$. Résolvons maintenant les inégalités

$$
\begin{gathered}
\frac{\beta(\beta-1)}{\mu \beta-1} \leqslant\|x+y\| \leqslant \beta(\mu-\beta)=k \\
\frac{\beta(\beta-1)}{\mu \beta-1} \leqslant\|x+y\| \leqslant 1=k^{\prime} .
\end{gathered}
$$

La seconde inégalité provient de la Proposition 13. On trouve en résolvant pour $\mu$ que, dans le premier cas,

$$
\mu \geqslant \frac{1+\beta^{2}+\sqrt{\left(1+\beta^{2}\right)^{2}-4 \beta}}{2 \beta}=\mu_{k}
$$

et que, dans le second cas,

$$
\mu \geqslant \frac{\beta^{2}-\beta+1}{\beta}=\mu_{k^{\prime}} .
$$

In n'est pas difficile de vérifier que, pour $1 \leqslant \beta \leqslant \sqrt{2}$, on a $\mu_{k} \geqslant \mu_{k^{\prime}}$. Donc, en se basant sur ce qui a été dit ci-dessus, on peut conclure que $k \leqslant k^{\prime}$ ou, en d'autres mots, que $\beta(\beta-\mu) \leqslant 1$. Donc

$$
\mu-\beta \leqslant \frac{1}{\beta}, \quad 1 \leqslant \beta \leqslant \sqrt{2}
$$

Montrons maintenant que $\mu-\beta \leqslant \beta / 2$, pour $\sqrt{2} \leqslant \beta \leqslant 2$. Puisque $\beta \geqslant \sqrt{2}$, il n'y a alors qu'un seul cas à considérer. Posons

$$
k \geqslant \frac{2(\beta-1)}{2 \mu-\beta}
$$


et résolvons pour $\mu$. On a alors

$$
\mu \geqslant \frac{\beta-1}{k}+\frac{\beta}{2}
$$

Posons

$$
\mu_{k}=\frac{\beta-1}{k}+\frac{\beta}{2}
$$

Si $\mu_{k} \geqslant \mu_{k^{\prime}}$ il est possible alors de conclure que $k \leqslant k^{\prime}$. Résolvons maintenant les inégalités

$$
\begin{gathered}
\frac{2(\beta-1)}{2 \mu-\beta} \leqslant\|x+y\| \leqslant \frac{2}{\beta}(\mu-\beta)=k \\
\frac{2(\beta-1)}{2 \mu-\beta} \leqslant\|x+y\| \leqslant 1=k^{\prime} .
\end{gathered}
$$

La seconde inégalité provient de la Proposition 13. On trouve en résolvant pour $\mu$ que, dans le premier cas,

$$
\mu \geqslant \frac{3 \beta+\sqrt{9 \beta^{2}-8 \beta}}{4}=\mu_{k}
$$

et que, dans le second cas,

$$
\mu \geqslant \beta-1+\frac{\beta}{2}=\frac{3}{2} \beta-1=\mu_{k^{\prime}} .
$$

Il n'est pas difficile de vérifier que, pour $\sqrt{2} \leqslant \beta \leqslant 2$, on a $\mu_{k} \geqslant \mu_{k^{\prime}}$. Donc, en se basant sur ce qui a été dit ci-dessus, on peut conclure que $k \leqslant k^{\prime}$ ou, en d'autres mots, que $2 / \beta(\beta-\mu) \leqslant 1$. Donc

$$
\mu-\beta \leqslant \frac{\beta}{2}, \quad \sqrt{2} \leqslant \beta \leqslant 2 .
$$

En résumé, nous venons de prouver que $\mu$ est dominé par le majorant

$$
\mu \leqslant \begin{cases}\beta+\frac{1}{\beta}, & \text { si } 1 \leqslant \beta \leqslant \sqrt{2} \\ \frac{3}{2} \beta, & \text { si } \sqrt{2} \leqslant \beta \leqslant 2\end{cases}
$$

ce qui est une amélioration par rapport au majorant $(\mu \leqslant \beta+1)$ donné par l'inégalité (1). 


\subsection{BORNES DE LA CONSTANTE RECTANGLE}

Nous pouvons combiner les résultats précédents dans une seule proposition:

Proposition 14. Soit $B$ un espace de Banach. Alors

et

$$
\begin{aligned}
& \mu \geqslant \begin{cases}\frac{1+\beta^{2}+\sqrt{\left(1+\beta^{2}\right)^{2}-4 \beta}}{2 \beta}, & \text { si } 1 \leqslant \beta \leqslant \frac{1+\sqrt{17}}{4} \\
2 \beta-1, & \text { si } \frac{1+\sqrt{17}}{4} \leqslant \beta \leqslant 2\end{cases} \\
& \mu \leqslant \begin{cases}\beta+\frac{1}{\beta}, & \text { si } 1 \leqslant \beta \leqslant \sqrt{2} \\
\frac{3}{2} \beta, & \text { si } \sqrt{2} \leqslant \beta \leqslant 2 .\end{cases}
\end{aligned}
$$

In est facile de montrer à partir de cette proposition que $\mu \leqslant \beta+1$. Ainsi le théorème de Baronti ([2], Théorème 3) est-il une conséquence directe de la Proposition 14 et la Proposition 13.1.

EXEMPLE 2. Kapoor et Mathur [16] ont donné les valeurs des paramètres $\beta\left(l_{3}^{2}\right)$ et $\beta\left(l_{4}^{2}\right)$ qui sont égaux à

$$
\begin{aligned}
& \beta\left(l_{3}^{2}\right)=\frac{1}{3} \sqrt[3]{17+7 \sqrt{7}} \approx 1.0957314 \\
& \beta\left(l_{4}^{2}\right)=\sqrt[4]{1+\frac{2}{3} \sqrt{3}} \approx 1.211565
\end{aligned}
$$

Par ailleurs, Gastinel et Joly [11] ont trouvé les valeurs correspondantes de $\mu$ qui sont:

$$
\mu\left(l_{3}^{2}\right) \approx 1.703 \quad \text { et } \quad \mu\left(l_{4}^{2}\right) \approx 1.888
$$

Vérifions dès lors le bien-fondé des bornes que nous donnons ci-dessus.

et

$$
1.703 \approx \mu\left(l_{3}^{2}\right) \leqslant \beta\left(l_{3}^{2}\right)+\frac{1}{\beta\left(l_{3}^{2}\right)} \approx 2.0083638
$$

$$
1.888 \approx \mu\left(l_{4}^{2}\right) \leqslant \beta\left(l_{4}^{2}\right)+\frac{1}{\beta\left(l_{4}^{2}\right)} \approx 2.0369437 .
$$

\section{MAJORANT DU BIAIS D'UN ESPACE UNIFORMÉMENT CONVEXE}

\subsection{CAS GÉNÉRAL}

Les résultats suivants sont fortement inspirés de [11]. Rappelons auparavant la définition suivante: 
DÉfinition 10: (Clarkson [4]) Soit $B$ un espace de Banach. $B$ est dit uniformément convexe, si, quels que soient $x$ et $y$ dans $B$ et $0<\varepsilon<2$, il existe une fonction $\delta(\varepsilon)>0$ telle que les conditions

$$
\|x\| \leqslant 1,\|y\| \leqslant 1,\|x-y\| \geqslant \varepsilon
$$

entraînent

$$
\left\|\frac{x+y}{2}\right\| \leqslant 1-\delta(\varepsilon) \text {. }
$$

Nous appellerons $\delta(\varepsilon)$ le module d'uniformité de $B$.

Soit donc $B$ un espace de Banach uniformément convexe de module d'uniformité $\delta(\varepsilon)$ et $x$ et $y$ deux vecteurs de $\mathcal{S}$. Appliquons le lemme 1.ii. On peut alors trouver un nombre réel $\gamma$ tel que

$$
x+\gamma y \perp_{B J} y .
$$

De la Proposition 4.1 on tire que $|\gamma| \leqslant 2$. Posons $\varepsilon=|\gamma|$. Puisque $x+\gamma y \perp_{B J} y$ alors, par définition, $\|x+\lambda y\| \geqslant\|x+\gamma y\|, \forall \lambda \in \mathbf{R}$. En posant $\lambda=0$ on trouve que

$$
\begin{aligned}
\|x+\gamma y\| & \leqslant\|x\| \\
& =1 .
\end{aligned}
$$

De plus

$$
\begin{aligned}
\|\boldsymbol{y} y\| & =|\gamma| \cdot\|y\| \\
& =|\gamma| \\
& =\varepsilon .
\end{aligned}
$$

Posons maintenant $X=x$ et $Y=x+\gamma y$. Alors

et

$$
\begin{aligned}
\|X\| & =\|x\|=1, \\
\|Y\| & =\|x+\gamma y\| \leqslant 1 \\
\|X-Y\| & =\|x-(x+\gamma y)\| \\
& =\|-\gamma y\| \\
& =|\gamma| \cdot\|y\| \\
& =|\gamma| \\
& =\varepsilon .
\end{aligned}
$$

Ce qui entraine que

$$
\Longrightarrow \quad \begin{array}{ll} 
& \left\|\frac{X+Y}{2}\right\| \leqslant 1-\delta(\varepsilon) \\
\left\|x+\frac{\gamma}{2} y\right\| \leqslant 1-\delta(\varepsilon) .
\end{array}
$$


Puisque

alors

et

$$
\begin{aligned}
\left\|x+\frac{\gamma}{2} y\right\| & \geqslant\|x+\gamma y\| \\
\|x+\gamma y\| & \leqslant 1-\delta(\varepsilon) \\
\|x+\gamma y\|+\delta(\varepsilon) & \leqslant 1 .
\end{aligned}
$$

Or

$$
\begin{aligned}
\|x+\gamma y\| & \geqslant\|\gamma y\|-\|x\| \\
& =|\gamma| \cdot\|y\|-\|x\| \\
& =|\gamma|-1 \\
|\gamma| & +\delta(\varepsilon) \leqslant 2
\end{aligned}
$$

c'est-à-dire que

$$
\varepsilon+\delta(\varepsilon) \leqslant 2 .
$$

On sait que la fonction $\varepsilon \mapsto \delta(\varepsilon)$ est une fonction croissante de l'intervalle $] 0,2]$ dans l'intervalle $[0,1]$. Il existe donc un nombre réel $\varepsilon_{0}$ tel que

$$
\varepsilon_{0}=\sup \{\varepsilon \in \mathbf{R} \mid \varepsilon+\delta(\varepsilon) \leqslant 2\}
$$

Par conséquent,

$$
\beta(\mathcal{B})=\sup \left\{\gamma \in R \mid x+\gamma y \perp_{B J} y ; x, y \in \mathcal{S}\right\} \leqslant \varepsilon_{0}
$$

car un nombre $\gamma$ tel que $x+\gamma y \perp_{B J} y$ (avec $x$ et $y$ dans $\mathcal{S}$ ) doit nécessairement satisfaire l'inégalité ci-dessus.

\subsection{Cas D'Un espace de Hilbert}

On sait [20] que si $B$ est un espace de Hilbert alors

$$
\delta(\varepsilon)=1-\sqrt{1-\frac{\varepsilon^{2}}{4}}
$$

pour $0 \leqslant \varepsilon \leqslant 2$. Remplaçons $\delta(\varepsilon)$ par sa valeur dans l'inégalité ci-dessus. Alors

$$
\varepsilon+1-\sqrt{1-\frac{\varepsilon^{2}}{4}} \leqslant 2 .
$$

En résolvant cette inégalité, on trouve que $\varepsilon \leqslant 8 / 5$ ou encore que $\beta(B) \leqslant 8 / 5$. En revanche, la Proposition 5 donne la meilleure valeur possible: $\beta(B)=1$. 


\subsection{Cas Des espaces $L^{p}, p \geqslant 2$}

Soit $p \geqslant 2$. On sait [12] que si $B=L^{p}$ alors

$$
\delta(\varepsilon)=1-\left(1-\left(\frac{\varepsilon}{2}\right)^{p}\right)^{\frac{1}{p}}
$$

avec $0 \leqslant \varepsilon \leqslant 2$. Substituons dans l'inégalité (4). On trouve que

$$
(\varepsilon-1)^{p}+\left(\frac{\varepsilon}{2}\right)^{p} \leqslant 1 .
$$

On peut borner supérieurement la solution de l'inégalité précédente en approximant la solution de l'équation

$$
(\varepsilon-1)^{p}+\left(\frac{\varepsilon}{2}\right)^{p}=1 .
$$

Ce qui entraine que, pour $p \geqslant 2$, on a

$$
\beta\left(L^{p}\right) \leqslant 2-\frac{2}{3 p} .
$$

Remarquons que l'inégalité donnée par Gastinel et Joly ([11], p.211) est une conséquence de l'inégalité ci-dessus car $\mu \leqslant \beta+1$ ([2], Théorème 3).

\subsection{CAS DES ESPACES $L^{p}, 1 \leqslant p \leqslant 2$}

Le résultat suivant, dû à Meir, est nécessaire pour majorer la constante $\beta\left(L^{p}\right)$ dans le cas $1 \leqslant p \leqslant 2$.

Proposition 15. (Meir [18], Corollaire, p.424) Soit $1<p \leqslant 2,0<\lambda<1$, $x, y \in L^{p}$ avec $\|x\| \leqslant 1,\|y\| \leqslant 1$ et $\|x-y\| \geqslant \varepsilon$. Alors

$$
\|\lambda x+(1-\lambda) y\| \leqslant 1-\frac{p-1}{4} m \varepsilon^{2},
$$

où $m=\min \{\lambda, 1-\lambda\}$.

Tout comme au paragraphe 5.1, posons $X=x$ et $Y=x+\gamma y$. Alors

et

$$
\begin{gathered}
\|X\|=\|x\|=1, \\
\|Y\|=\|x+\gamma y\| \leqslant 1 \\
\|X-Y\|=|\gamma|=\varepsilon .
\end{gathered}
$$

Ce qui entraîne que

$$
\begin{aligned}
& \|\lambda X+(1-\lambda) Y\| \leqslant 1-\frac{p-1}{4} m \varepsilon^{2} \\
& \|\quad\| x+(1-\lambda) \gamma y \| \leqslant 1-\frac{p-1}{4} m \varepsilon^{2} .
\end{aligned}
$$




\section{Puisque}

$$
\begin{array}{ll} 
& \|x+(1-\lambda) \gamma y\| \geqslant\|x+\gamma y\| \\
\text { alors } & \|x+\gamma y\| \leqslant 1-\frac{p-1}{4} m \varepsilon^{2} \\
\text { et } & \|x+\gamma y\|+\frac{p-1}{4} m \varepsilon^{2} \leqslant 1 .
\end{array}
$$

Or $\|x+\gamma y\| \geqslant|\gamma|-1$,

$\Longrightarrow$

c'est-à-dire que

ou encore que

$$
|\gamma|+\frac{p-1}{4} m \varepsilon^{2} \leqslant 2
$$

$$
\varepsilon+\frac{p-1}{4} m \varepsilon^{2} \leqslant 2
$$$$
\varepsilon+\frac{p-1}{4} m \varepsilon^{2}-2 \leqslant 0 .
$$

Il suffit maintenant de raisonner comme il a été fait au paragraphe 5.1. L'équation $\varepsilon+p-1 / 4 m \varepsilon^{2}-2=0$ ayant comme racines les expressions

$$
\begin{gathered}
r_{0}=\frac{2(\sqrt{1+2 m(p-1)}-1)}{m(p-1)}>0 \\
r_{1}=\frac{-2(\sqrt{1+2 m(p-1)}+1)}{m(p-1)}<0
\end{gathered}
$$

on obtient que $\varepsilon_{0}=\frac{2(\sqrt{1+2 m(p-1)}-1)}{m(p-1)}=\frac{4}{1+\sqrt{1+2 m(p-1)}}$.

Naturellement, cette expression 'est minimisée lorsque la valeur du paramètre $m$ est maximale, c'est-à-dire lorsque $\lambda=1 / 2$. Ce qui entraîne que, pour $1<p \leqslant 2$, on a

$$
\beta\left(L^{p}\right) \leqslant \frac{4}{1+\sqrt{p}}
$$

L'inégalité est encore vraie dans le cas $p=1$. En effet, la Proposition 4.5 affirme que $\beta\left(L^{1}\right)=2=4 / 1+\sqrt{1}$. En conclusion

$$
\beta\left(L^{p}\right) \leqslant \frac{4}{1+\sqrt{p}}, \quad 1 \leqslant p \leqslant 2 .
$$

En se servant de l'inégalité (1) on obtient le majorant de $\mu$ suivant:

$$
\mu\left(L^{p}\right) \leqslant \frac{5+\sqrt{p}}{1+\sqrt{p}}
$$


avec $1 \leqslant p \leqslant 2$. En résumé, nous avons trouvé que

et que

$$
\begin{aligned}
& \beta\left(L^{p}\right) \leqslant \begin{cases}\frac{4}{1+\sqrt{p}}, & \text { si } 1 \leqslant p \leqslant 2 \\
2-\frac{2}{3 p}, & \text { si } 2 \leqslant p<\infty .\end{cases} \\
& \mu\left(L^{p}\right) \leqslant \begin{cases}\frac{5+\sqrt{p}}{1+\sqrt{p}}, & \text { si } 1 \leqslant p \leqslant 2 \\
3-\frac{2}{3 p}, & \text { si } 2 \leqslant p<\infty .\end{cases}
\end{aligned}
$$

Ce dernier résultat étend celui de Gastinel et Joly ([11], p.211).

\section{REFERENCES}

[1] A.M. Al-Rashed, 'Norm inequalities and characterizations of inner product spaces', Amer. Math. Soc. (submitted).

[2] M. Baronti, 'Su alcuni parametri degli spazi normati', Boll. Un. Mat. Ital. B (5) 18 (1981), 1065-1085.

[3] G. Birkhoff, 'Orthogonality in linear metric spaces', Duke Math. J. 1 (1935), 169-172.

[4] J.A. Clarkson, 'Uniformly convex spaces', Trans. Amer. Math. Soc. 40 (1936), 396-414.

[5] D.G. DeFigueiredo and L.A. Karlovitz, 'On the radial projection in normed spaces', Bull. Amer. Math. Soc. 73 (1967), 364-368.

[6] M. Del Rio and C. Benitez, 'The rectangular constant for two-dimensional spaces', $J$. Approx. Theory 19 (1977), 15-21.

[7] J. Desbiens, 'Sur le biais d'un espace de Banach', Ann. Sci. Math. Québec (to appear).

[8] C.F. Dunkl and K.S. Williams, 'A simple norm inequality', Amer. Math. Monthly 71 (1964), 53-54.

[9] R. Fortet, 'Remarques sur les espaces uniformément convexes', Bull. Soc. Math. France 67-69 (1939-41), 23-46.

[10] C. Franchetti, 'On the radial constant of real normed spaces', in Approximation Theory III, pp. 425-428 (Academic Press, New York, 1980).

[11] N. Gastinel and J.L. Joly, 'Condition numbers and general projection method', Linear Algebra Appl. 3 (1970), 185-224.

[12] O. Hanner, 'On the uniform convexity of $L^{p}$ and $l^{p}$ ', Ark. Mat 3 (1955), 239-244.

[13] R.C. James, 'Orthogonality and linear functionals in normed linear spaces', Trans. Amer. Math. Soc. 61 (1947), 265-292.

[14] R.C. James, 'Uniformly non-square banach spaces', Ann. of Math. 80 (1964), 542-550.

[15] J.L. Joly, 'Caractérisation d'espace hilbertien au moyen de la constante rectangle', $J$. Approx. Theory 2 (1969), 301-311.

[16] O.P. Kapoor and S.B. Mathur, 'Metric Projection Bound and the Lipschitz Constant of the Radial Retraction', J. Approx. Theory 38 (1983), 66-70.

[17] J.L. Massera and J.J. Schäffer, 'Linear differential equations and functional analysis', Ann. of Math. 67 (1958), p. 538. 
[18] A. Meir, 'On the uniform convexity of $L^{p}$ spaces, $1<p \leqslant 2$ ', Illinois J. Math. 25 (1984), 420-424.

[19] C. Morales, 'Pseudo-contractive mappings and the Leray-Schauder boundary conditions', Comment. Math. Univ. Carolin. 20 (1979), 745-756.

[20] G. Nordlander, 'The modulus of convexity in normed linear spaces', Ark. Mat. 4 (1958), 15-17.

[21] M.A. Smith, 'On the Norms of Metric Projections', J. Approx. Theory 31 (1981), 224-229.

[22] R.L. Thele, 'Some results on the radial projection in Banach spaces', Proc. Amer. Math. Soc. 42 (1974), 483-486.

Département d'informstique

Collège Militaire Royal de Saint-Jean

Saint-Jean-sur-Richelieu

Québec, Canada, J0J 1R0 